\title{
Micro-política de una escuela marginalizada entre inclusión y estandarización
}

\author{
Micro-politics of a marginalized school: \\ between inclusion and standardization
}

\author{
Muriel Armijo Cabrera \\ Universidad Alberto Hurtado-Universidad Diego Portales-Université Paris 8 \\ murielarmijo.c@gmail.com \\ Código Orcid: http://orcid.org/0000-0003-1657-6453
}

\begin{abstract}
Resumen
En el marco de la Ley de Inclusión (20.845/2015) que promueve la mixtura social de los estudiantes chilenos, se indaga una escuela subvencionada en contexto de pobreza que ha promovido esta mixtura desde su creación en 1980, al ser gratuita y sin selección. Se busca entender cómo se despliegan los procesos de inclusión/exclusión social de las niñas y los niños en este escenario de marginalidad. ¿Qué significa entonces "incluir"? ¿Cuáles son las fronteras entre inclusión y exclusión social en la escuela? Se realiza una etnografía escolar interpretativa durante siete meses meses, con un foco en un curso de $4^{\circ}$ básico. Los primeros hallazgos de campo se centran en la micro-política de la escuela (Ball, 1987). Se identifican tres dispositivos principales: un dispositivo identitario, que descansa en el ethos histórico de la escuela y su proyecto de inclusión social; un dispositivo tradicional, que recoge las trayectorias culturales de los sujetos y las representaciones presentes en la sociedad; un dispositivo tecnocrático, que se instala progresivamente desde el sistema educativo en pos de una estandarización del proceso educativo. Durante el proceso investigativo, se observa la evolución del equilibrio entre los tres dispositivos en la escuela, dónde las finalidades éticas de inclusión están superadas por los objetivos tecnocráticos de estandarización. Se evidencian prácticas pedagógicas cooperativas existentes en la escuela que van desapareciendo en beneficio de tecnologías de clasificación y estrategias competitivas.
\end{abstract}

\section{Palabras clave}

Exclusión social, estandarización, educación básica, pobreza, cooperación, competencia.

Forma sugerida de citar: Armijo Cabrera, Muriel (2019). Micro-política de una escuela marginalizada: entre inclusión y estandarización. Universitas, 20, pp. 191-209. 


\begin{abstract}
Considering the Law of Inclusion (20.845/2015), which promotes social mixture of Chilean students, this research studies a subsidized school in a context of poverty that has promoted this mixture since its creation in 1980, being free and without selection. We seek to understand how do the social inclusion/ exclusion processes of children deploy in this marginalized scene. What means then "to include"? Which are the frontiers between inclusion and exclusion in this school? We realized an interpretative school ethnography for 7 months, with a focus on a $4^{\text {th }}$ grade. The first results in the field are centered on the micropolitics of the school (Ball, 1987). We identify three principal dispositives: an identity dispositive that lies on the historical ethos of the school and its social inclusion project; a traditional dispositive, which reflects the cultural trajectories of the subjects and the representations presents in society; a technocratic dispositive that is gradually installed from the educative system looking for the standardization of the educative process. During the research process, we observe the evolution of the equilibrium between the three dispositives in the school, where the ethical finalities of inclusion are overcome by the technocratic objectives of standardization. We present cooperative pedagogical practices existing in the school that disappear to the benefit of classification technologies and competitive strategies.
\end{abstract}

Keywords

Social exclusion, standardisation, basic education, poverty, cooperation, competition.

\title{
Introducción
}

En Chile, se ha promulgado la Ley de Inclusión (20.845/2015) que busca promover de la mixtura social de los estudiantes, mediante la prohibición del lucro, del financiamiento compartido con las familias, y de la selección de los estudiantes en los establecimientos subvencionados por el Estado. Estos representan un $92 \%$ de los establecimientos de enseñanza obligatoria, repartidos entre municipales (37\%) y particulares subvencionados $(55 \%)$. Durante la dictadura cívico-militar, el sistema escolar chileno es privatizado (Decreto con Fuerza de Ley $N^{\circ} 1-3063 / 80$; Decreto Ley 3.476/80) y regulado por la demanda educativa o voucher, subsidio público entregado a los establecimientos particulares y municipales. Después de la dictadura, la ley de financiamiento compartido de 1993 permite multiplicar los recursos educativos solicitando la participación de las familias (Valenzuela, Bellei \& De los Ríos, 2014), lo que lleva a una progresiva segmentación del sistema, según el nivel de pago exigido. Se delega la responsabilidad de la educación 
de las niñas y niños chilenos a sus familias, cuya "libertad de enseñanza" se configura como un pilar de la Constitución de 1980. La ley de oferta y demanda así estimulada permite el despliegue del cuasi-mercado educativo (Slachevsky, 2015). En este escenario, la Ley de Inclusión pretende reducir las barreras en el acceso a los establecimientos escolares, al prohibir las prácticas de selección socio-económica y cultural.

El sistema educativo chileno constituye una máquina de estratificación que fragmenta la sociedad según sus recursos socio-económicos (Bellei, 2013). La educación se concibe como un bien de consumo privado, que se puede adquirir con el esfuerzo individual, lo que condiciona la elección de escuela (Rojas, Falabella \& Leyton, 2016). Las familias despliegan entonces estrategias para elegir su escuela y maximizar las oportunidades de sus hijas e hijos, distinguiéndose en función de sus ingresos y contribuyendo así en segmentar el sistema educativo en estratos socio-económicos. La Ley de Inclusión introduce un cuestionamiento de la segmentación socio-económica, al privilegiar una perspectiva sobre inclusión centrada en la mixtura social de los estudiantes (Rojas \& Armijo, 2016). Es impulsada por el movimiento social estudiantil que emerge en 2006 con la llamada "Revolución pingüina" y las discusiones sobre calidad y equidad de la educación que se despliegan desde entonces. Se diseña un nuevo sistema de admisión que se empieza a implementar en 2016. Este reparte de manera aleatoria los estudiantes, previa postulación de las familias, para impedir el uso de discriminaciones arbitrarias que puedan limitar el acceso de los estudiantes a los establecimientos.

En este contexto, la presente investigación se ha centrado en una escuela que nunca ha aplicado ni financiamiento compartido ni selección de estudiantes, desde sus inicios, al alero de las políticas de privatización de la educación. Surge en un contexto de marginalización extrema, en una zona periférica de Santiago que se encontraba entonces en un proceso de urbanización controlado por las políticas de "homogeneización social" de la dictadura (Morales \& Rojas, 1986). Estas políticas desplazan e instalan a familias pobres provenientes de otros sectores de la Región metropolitana, conformando territorios marginalizados en sectores todavía rurales. Las condiciones de habitación y de higiene son precarias, los nuevos pobladores carecen de medios de transporte, equipamientos educativos, de salud y comercios (Ducci, 1997; Gurovich, 1999). Se estudia una escuela básica que nace con un proyecto político y pedagógico, orientándose a la inclusión social. Enfoca su atención en los estudiantes más marginalizados del sistema educativo, excluidos de otros esta- 
blecimientos por distintos motivos: resultados, comportamiento, discriminaciones o socio-culturales étnicas. Hoy en día, el sector donde se ubica sigue marginalizado, en los planes socioeconómico, geográfico y cultural. Según el "Índice de Vulnerabilidad Escolar" elaborado en Chile, tiene un 90\% de vulnerabilidad. ¿Qué significa "incluir" en este contexto? ¿Cuáles son las fronteras entre inclusión y exclusión en una escuela marginalizada?

\section{Marco teórico}

Esta investigación se enmarca en la perspectiva post-estructuralista, asumiendo el "giro ontológico" que cuestiona las distinciones binarias entre sujeto y objeto, mente y cuerpo, naturaleza y cultura, entre muchas otras (Adams St. Pierre, 2014; Adams St. Pierre, Jackson \& Mazzei, 2016). Se concibe la inclusión/exclusión social como un proceso dinámico, que produce de manera permanente el campo social (Armijo-Cabrera, 2018). La inclusión/exclusión social se genera en las interrelaciones entre los sujetos, produciendo diferencias e identidades, al identificar a sus "otros" significativos. Desde esta perspectiva, la inclusión implica la determinación de un colectivo limitado por fronteras, que surgen en función de criterios socio-históricos variables y situados (Lindblad \& Popkewitz, 2001; Graham \& Slee, 2008). A partir de la lectura de Michel Foucault, se asume que los modos de constitución del sujeto se vinculan estrechamente con relaciones de poder y con producciones de saberes (Ball, 2012). Así, la inclusión/exclusión social consiste en un proceso permanente de definición del centro "normal", un lugar de poder que excluye a los que pretende incluir (Matus \& Infante, 2011). Este se constituye a través de saberes y reglas que se aplican sobre los cuerpos. Se considera así que el proceso de inclusión/exclusión social se expresa en las subjetivaciones de los actores, en el momento en que elaboran identidades y diferencias, las que se inscriben en su corporalidad. Estos procesos están implicados en sistemas de poder que producen subjetividades desiguales en base distintos marcadores reforzados por el sistema escolar: clase, raza, género, entre otros (Apablaza, 2015; Walsh, 2012; Dussel, 2004; de la Rosa, 2015).

En efecto, la escuela se conforma como un espacio social de menor escala, inserto en un entorno social y cultural específico. En ese sentido, los procesos de inclusión/exclusión social se dan de la misma manera en su interior, con normas y regulaciones propias. Además, en el marco de una or- 
ganización escolar, donde se divide y se jerarquiza el trabajo, se despliegan relaciones de control y conflictos (Ball, 1987). El poder se aplica localmente a través de los dispositivos, que contiene dimensiones discursivas y extradiscursivas. Los dispositivos provienen del entorno, son apropiados y tensionados en sus puntos de resistencia dentro de la escuela (Foucault, 1982). Los sujetos escolares se desenvuelven entonces en una organización limitada pero permeable a sus entornos, tanto político como socio-cultural. En función de sus valores y de su lugar en la organización, los distintos sujetos escolares interpretan el mundo y le dan sentido, mediante discursos y prácticas. El juego de los intereses y de las subjetividades conforma de manera cotidiana lo que Stephen Ball ha denominado la "micro-política" de la escuela (Ball, 1987). Esta aparece en las disputas entre distintas perspectivas y maneras de actuar dentro de la escuela, que tensionan a los sujetos.

El objetivo general de esta investigación es entender cómo se despliegan los procesos de inclusión/exclusión social de los niños en una escuela ubicada en un contexto de alta marginalidad. En este artículo se presentan los resultados del primer objetivo específico: Identificar las dinámicas y los dispositivos escolares que afectan los procesos de inclusión/exclusión social de los niños en la escuela. En ese sentido, estos hallazgos parciales se centran en la experiencia y el juego de los adultos, que configuran la micro-política de la escuela.

\section{Metodología}

Se privilegia una metodología de investigación cualitativa de corte interpretativo, acorde con el marco teórico post-estructuralista, donde quien investiga está inmerso en su objeto de estudio, es decir la escuela descrita anteriormente como caso de estudio particular. Objetos y sujetos están interrelacionados en el proceso de la investigación, que viene a irrumpir la cotidianeidad escolar. En ese sentido, se incorporan los significados producidos por los sujetos mediante la experiencia de quien investiga. Las percepciones, sensaciones y emociones de quien investiga permiten acceder a la producción social que se realiza en las interacciones de la experiencia cotidiana (Adams St. Pierre, 1997; Blackman, 2007). La validez de este enfoque reside en la fidelidad a la experiencia, buscando ser "persuasiva y creíble" cuando relata las historias de los participantes, "representar sus pensamientos y sus experiencias" de manera fidedigna (Gallant, 2008, p. 247). 
Se ha realizado una etnografía escolar interpretativa (Guber, 2011) y visual (Pink, 2001), en la escuela mencionada. Esta se despliega durante siete meses, con un foco que se desplaza progresivamente desde el mundo adulto hacia las experiencias de las niñas y los niños. La investigación etnográfica implica un trabajo de campo que consiste en:

- La documentación del objeto de estudio (previo, durante y posterior al terreno).

- La observación participante en la escuela (centrada en un curso de $4^{\circ}$ básico).

- Entrevistas etnográficas con adultos y niños.

- Y por último una producción visual infantil (fotografías y dibujos), que fue asociada con entrevistas grupales de niños.

Se han registrado cotidianamente los diversos datos producidos en los diarios de campo y se han grabado y transcrito integralmente las entrevistas realizadas con los niños. Los datos se han analizados continuamente y reiteradamente en el propio trabajo de la escritura y a partir de matrices conceptuales (Adams St. Pierre, 2017). Estas fueron elaboradas de acuerdo al marco teórico, y evolucionaron durante la investigación, con los intercambios académicos y el diálogo con los autores que generan el trabajo conceptual (Rockwell, 2009). Los resultados fueron discutidos en instancias de reflexión académica variadas, buscando alcanzar una "subjetividad disciplinada" (Erickson, 1984; Serra, 2004). Más allá de la "triangulación" desarrollada en otros enfoques, al multiplicar los puntos de vista se llega a la "cristalización" los datos, como lo plantea Laurel Richardson (2000, pp.13-14):

I propose the central imaginary for validity for postmodernist texts is not the triangle-a rigid, fixed, two-dimensional object. Rather, the central imaginary is the crystal, which combines symmetry and substance with an infinite variety of shapes, substances, transmutations, multidimensionalities, and angles of approach. ${ }^{1}$

1 "Planteo que el imaginario central para la validez de los textos postmodernos no es el triángulo -un objeto rígido, fijo, en dos dimensiones. El imaginario central es más bien el cristal, que combina la simetría y la substancia con una variedad infinita de formas, substancias, transmutaciones, multidimensionalidades, y ángulos de aproximación.” (Traducción personal) 


\section{Análisis y resultados}

\section{Una escuela marginalizada en mutación}

Los primeros hallazgos de la investigación permiten describir y analizar la micro-política de la escuela (Ball, 1987), que se expresa en las disputas entre distintas visiones y maneras de actuar privilegiadas por los sujetos escolares. La escuela "Estrella" fue creada en 1980, en el mismo momento en que se abre el sistema escolar al mercado. Surge desde una oportunidad de emprendimiento como una iniciativa social, para responder a las necesidades educativas de una comunidad marginalizada en una periferia de Santiago en plena urbanización. El proyecto inicial de la escuela "Estrella" tenía la finalidad política, mediante la acción social, de educar a los más pobres que habían sido marginalizados. Se crea en una comuna del sur de Santiago, a proximidad de una toma de terreno histórica, a partir de una investigación colaborativa con los pobladores para identificar sus necesidades. Nunca ha adherido al financiamiento compartido ni ha seleccionado a sus estudiantes, por lo contrario, atendía "de preferencia, a los niños de hogares pobres discriminados por razones sociales, pedagógicas, conductuales o raciales". Según el Índice de Vulnerabilidad Escolar (IVE) chileno, elaborado por la JUNAEB (Junta Nacional de Auxilios Educativos y Becas), la escuela "Estrella" tiene un $90 \%$ de vulnerabilidad. Entre los establecimientos que tampoco aplican co-pago ni selección de estudiantes, tiene la tasa de vulnerabilidad más alta del sector. Durante los años 1980 y 1990, su matrícula aumenta y se asocia con otras iniciativas educativas y formativas. Destaca por ser un establecimiento declarado laico, en un contexto donde la educación privada estaba dominada por el mundo religioso (escuelas cristianas y parroquiales). Esta escuela se dirige a los estudiantes de educación básica y parvularia, desde pre-Kinder hasta $8^{\circ}$ básico, y recibe en la actualidad aproximadamente 350 estudiantes.

La escuela "Estrella" considera las particularidades de su tejido urbano por lo que se inscribe en la red de instituciones colaboradoras del SENAME (Servicio Nacional de Menores), organismo gubernamental dependiente del Ministerio de Justicia chileno. Beneficia de una subvención específica desde 1991 con dos dispositivos orientados hacia la población más marginalizada: por un lado recibe estudiantes retirados de sus familias, residentes de hoga- 
res del SENAME o en procesos de judicialización; por otro lado, desarrolla desde 1990 un Centro Abierto Diurno (CAD), que se convierte en 2004 en Centro Comunitario por los derechos Infanto-Juveniles (CCIJ), organismo del SENAME que busca prevenir de manera comunitaria la "vulneración de derechos", a través de "la implementación de proyectos de aprendizaje de carácter grupal" (SENAME, 2004, citado por Silva \& Gallardo, 2006, p. 3). Además la escuela constituye un Programa de Integración Escolar (PIE) desde 2004 dirigido a atender los estudiantes con "necesidades educativas especiales" (NEE) permanentes o transitorias. En 2015 se cuentan 63 estudiantes en el Programa es decir un 19\% de los estudiantes de la escuela. La escuela también adhiere a la Subvención Escolar Preferencial (SEP) desde su lanzamiento en 2008, beneficiando así de una subvención adicional para todos los niños considerados vulnerables. Además es una escuela con una componente multicultural importante para el sector, puesto que en 2015 se declaraban 48 estudiantes pertenecientes a la etnia Mapuche, es decir un $12 \%$ del alumnado.

Durante las primeras décadas, se constituye un colectivo profesional que permanece hasta los años 2010, desarrollando prácticas pedagógicas cooperativas y participativas. Con la desaparición del fundador, el equipo se empieza a quebrar generando una reconfiguración de la dinámica interna de la escuela. Se mencionan dos olas de despidos masivos, en 2013 y 2016, que conducen a una renovación importante del plantel docente. Los nuevos integrantes no son capacitados para ejercer las técnicas pedagógicas cooperativas, debilitando el funcionamiento innovador de la escuela. En 2017, la escuela recibe la visita de la Agencia de la Calidad y la auditoría de la Superintendencia, nuevos organismos del Estado centrados en el Aseguramiento de la Calidad de la Educación (Ley SNAC, 2011). La escuela "Estrella" se compromete con inversiones de infraestructura, revisa sus indicadores de gestión y de calidad, se ve presionada para mejorar sus resultados en las pruebas nacionales estandarizadas (Sistema de Medición de la Calidad Educativa, en adelante, SIMCE).

\section{Una escuela tensionada entre tres dispositivos}

En este escenario, se identifican tres dispositivos principales que atraviesan la escuela y la tensionan: un dispositivo identitario, que descansa en 
el ethos histórico de la escuela y su proyecto de inclusión social; un dispositivo tradicional, que recoge las trayectorias culturales de los sujetos y las representaciones presentes en la sociedad; un dispositivo tecnocrático, que se instala progresivamente desde el sistema educativo en pos de una estandarización del proceso educativo. Estos tres dispositivos resultan del análisis realizado de manera continua en el campo y del análisis dialógico producido en los intercambios académicos y bibliográficos. Sus denominaciones han sido objeto de reflexión y de discusión.

\section{Dispositivo identitario}

Se relaciona con el proyecto social de la escuela, que surge en un contexto de resistencia a la dictadura. El fundador era un maestro normalista que concebía la educación como una vía de emancipación, en la línea de la Reforma educacional de 1965 y con la influencia de pensadores como Paulo Freire y su Pedagogía del oprimido (1970). Exonerado por la dictadura, el fundador desarrolla proyectos que le permiten perseguir su labor educativa y transformadora. Se centra en los territorios con más necesidad, dedicándose a la educación de los más pobres. Desde esta línea pedagógica, la educación en contextos marginalizados debe pasar por dispositivos pedagógicos diseñados para movilizar a los sujetos, favoreciendo su participación para suscitar el sentido de la experiencia escolar y la relevancia de los contenidos de aprendizaje. Se proponen actividades en relación con el entorno, en particular la producción de un huerto y de una granja escolar en este sector todavía rural; instancias participativas para niños y adultos, con asambleas semanales y consejos escolares que permiten regular la disciplina; trabajo cooperativo y creativo, mediante distintas herramientas pedagógicas (Freire, 1970).

Este dispositivo dibuja un discurso específico sobre inclusión/exclusión social, donde los límites entre identidades y diferencias están determinados por esta visión de la pobreza que es su foco de atención. Los niños son los "otros" de los adultos, son los pobres por los cuales se involucran en una acción social. Se superponen así las fronteras etarias entre adultos y niños, con las fronteras socio-culturales entre pobres y menos pobres. En efecto, los adultos que trabajan en la escuela tampoco se definen como privilegiados económicamente, pero rechazan la pobreza específica de los niños, que se concibe en un sentido amplio. En la actualidad, la imagen de la pobreza está reforzada por la idea de "vulnerabilidad", proveniente de los dispositivos tecnocráticos, que determinan y calculan los "índices de vulnerabilidad". Se 
entiende como una pobreza multidimensional, que genera "riesgos" de caer en la pobreza para los sujetos. Esta noción de vulnerabilidad se observa en las experiencias de los sujetos, a través de los casos de situaciones familiares relatadas durante el terreno: violencias, abandonos, adicciones, detenciones, abusos sexuales (ver escena 1).

Escena 1: Relato de un caso de abuso y violencia familiar:

La profesora dice que estos niños con todos los problemas que tienen en la casa no están disponibles para "aprender matemática". Me cuenta más historias familiares de los niños: la asistente social estaba muy afectada el otro día por el caso de un niño de $1^{\circ}$, secuestrado y abusado por su padre durante una semana, luego sacado de la familia vecina que se hacía cargo de él y que había logrado sacarle la información necesaria para poder inculpar al padre, para alejarlo. Entonces el chico ahora estaba en un hogar, solo, y no había nada que hacerle (Diario de campo, 05/04/2017).

Las condiciones de vulnerabilidad del entorno social, que determinan al estudiantado, movilizan los afectos de los adultos en la escuela que generan un compromiso con "sus" niños. Los docentes y asistentes de la educación se ven enfrentados de manera cotidiana con situaciones de violencia extrema, las que alimentan este discurso identitario sobre inclusión/exclusión social, donde los "otros" son los niños, los pobres.

\section{Dispositivo tradicional}

Se configura como un zócalo de base de la cultura escolar, anclado en el entorno social y cultural. Proviene de las trayectorias personales de los sujetos, inmersos en una sociedad marcada por las desigualdades y por la religión. Los adultos que trabajan en la escuela comparten el mismo nivel socio-económico que los niños, clases medias bajas, primeros universitarios de su familia o herederos de familias de maestros. Algunos incluso habitan el mismo territorio, vecinos y portadores de la identidad barrial. Además, las condiciones laborales de los docentes y de los otros trabajadores de la escuela les confieren un estatus precario. El dispositivo tradicional consiste entonces en una repetición de las normas sociales observadas en el entorno y practicadas en la experiencia cotidiana. En particular se observa en las prácticas pedagógicas tradicionales y autoritarias, que se despliegan en la escuela: orden del aula en filas de bancos y sillas alineados frente a la pizarra; clases expositivas del maestro copiadas en silencio por los niños y ejer- 
cicios de aplicación de contenido o guías corregidos por el maestro; reglas de vestimenta con uniforme diferenciado y formación de los cursos por género (filas de "señoritas y caballeros").

El dispositivo tradicional dibuja entonces un discurso sobre inclusión/ exclusión social que reproduce las desigualdades que atraviesan esa sociedad compartida. Tal como lo plantean Lindblad y Popkewitz (2001, p. 4): "the relation of governance to social inclusion and exclusion embodies a configuration of cultural, economy and social struggles". ${ }^{2}$ Se marcan las diferencias etarias a través de los modales autoritarios, se incorpora la religiosidad en las interacciones cotidianas, se performan los estereotipos de género. En particular se observa el clasismo y la reproducción de las categorías socio-económicas, cuando una adulta le contesta a un niño "no me hables así, yo no soy tu nana". También se observa la repetición del nacionalismo y del patriotismo promovido por el entorno tanto político como social, cuando por ejemplo se canta el himno nacional "como buenos chilenos". Existe un equipo de futbol femenino, pero es marginado en los encuentros deportivos organizados en la escuela, denotando el sexismo y la discriminación de género. Por último, el tema de la homosexualidad se elude en la escuela cuando surge desde los niños, genera incomodidad y queda "en manos de Dios".

\section{Dispositivo tecnocrático}

Se instala con el giro de los años 2000 y 2010, al alero de las políticas educativas consiguientes a la "Revolución pingüina". Si la liberalización y la mercantilización de la educación se establecen en dictadura, los dispositivos de evaluación y control que amarran el sistema se elaboran casi tres décadas después (Falabella, 2015). En particular se despliega a partir de cuatro normativas: la Ley SEP (2008) que instaura la rendición de cuentas; el Decreto 170 (2009) que determina los procedimientos para diagnosticar "necesidades educativas especiales"; la Ley General de Educación (LGE, 2009) que fija las responsabilidades del Estado evaluador; y la Ley sobre el Sistema Nacional de Aseguramiento de la Calidad (SNAC, 2011) que define las atribuciones de cada instancia nuevamente creada, entre ellas la Agencia de la Calidad y la Superintendencia. El dispositivo tecnocrático viene a tensionar con fuerza el dispositivo identitario de la escuela, surgido en otro con-

2 "La relación de la gobernanza con la inclusión y exclusión social encarna una configuración de luchas culturales, económicas y sociales” (Traducción personal). 
texto histórico y político. Se observa en particular durante el terreno en las relaciones con la Agencia de la Calidad que visita y evalúa a la escuela. Los sujetos escolares resisten sus críticas, pero a su vez incorporan los criterios de evaluación que se les imponen desde arriba.

El dispositivo tecnocrático dibuja un discurso sobre inclusión/exclusión social que categoriza a los sujetos entre normales y anormales, en base a criterios considerados "objetivos". Estos se despliegan a través de sistemas de evaluación y de medición, mediante herramientas pedagógicas conductistas y estandarizadas (Casassus, 2010). Estos criterios descansan en el diagnóstico y la clasificación de los sujetos según categorías rígidas y estáticas como las "necesidades educativas especiales", las que son validadas por pruebas psicométricas y aplicadas por profesionales específicos (Peña, 2013). Definen así una noción de "normalidad" de manera externa, a través de normativas y evaluaciones, que determinan un "estándar" objetivo y objetivable (Matus \& Infante, 2011). Desde este discurso, los estudiantes deben competir para alcanzar los estándares de manera individual, los que no cumplan, considerados "anormales", son excluidos en los márgenes escolares y sociales. En la escuela "Estrella", por ejemplo, los docentes trabajan de la mano con los profesionales psico-sociales, que despliegan estrategias de evaluación psicométricas y conductistas, determinando decisiones pedagógicas (ver escena 2).

Escena 2: La exclusión de los niños desde una lectura conductista del aprendizaje:

Finalmente, la psicóloga habla del paseo de curso, que todavía faltan muchas autorizaciones para la próxima salida el día 22 , y que entonces ella piensa que no hay que llevárselos no más, porque si no es "fomentar la conducta" (Diario de Campo, 12/06/2017).

\section{Una escuela cooperativa que deviene competitiva}

Cada uno de estos dispositivos atraviesa la escuela y la tensiona. Convergen en algunos aspectos, también se oponen, como lo muestra la tensión entre la identidad histórica de la escuela y las evoluciones del sistema educativo reflejadas en el dispositivo tecnocrático. Los tres dispositivos aparecen en los discursos y las prácticas de los distintos sujetos sin pertenecerles. Se observan diferencias generacionales entre los adultos, con discursos más 
sociales o más competitivos, pero no son suficientes para atribuirlos a un grupo etario. De la misma manera, los discursos desplegados por los profesionales de la salud, y sus herramientas de trabajo, performan el dispositivo tecnocrático, empero no les impide compartir otros discursos. Estos tres dispositivos ofrecen una posibilidad de interpretación del mundo y un marco de decisión para la acción. Los sujetos se los apropian, los utilizan y traducen, pero también les resisten, los discuten y los escapan (Ball, 1987).

Durante el proceso de campo, se observa la evolución del equilibrio entre los tres dispositivos, donde las finalidades éticas de inclusión son superadas por los objetivos tecnocráticos de estandarización. Al principio del año, una docente encargada de $4^{\circ}$ básico acepta recibir la investigación, pese a la prueba del SIMCE aplicada al final de ese año, considerando que es "una evaluación más". Una directiva les aclara a los niños que lo más importante para ellos este año es "ser feliz", no el SIMCE. Se evidencia, en estos primeros momentos del año escolar, la preeminencia del dispositivo identitario, centrado en el desarrollo personal de los niños. No obstante, avanzando el año, el SIMCE cobra cada vez más importancia en los discursos y las prácticas. Surge en las reuniones como una presión que recae en la profesora jefa; se comentan los resultados del año anterior; se multiplican los ensayos SIMCE. Para maximizar los esfuerzos, los ensayos SIMCE son monitoreados y corregidos por el equipo directivo. Toda la energía de la escuela converge y se orienta progresivamente hacia su realización (ver escena 3).

Escena 3: La tensión generada por las pruebas estandarizadas en una reunión de ciclo:

Dice que tiene muchas dificultades, que le ponen la presión con el SIMCE, que hay un problema con el nivel de los niños. Le dicen que no tiene que estar ansiosa, pero Elvira contesta que obvio que está ansiosa, porque ella tiene la presión por el SIMCE, que siente que trabaja en contra del tiempo. Dice que a ella le dijeron que no se trabajaba para el SIMCE, y ahora sí. Las otras dicen que debería haber una reflexión específica sobre el SIMCE, la UTP dice que tiene que hacerse antes del fin de semestre (Diario de Campo, 12/06/2017).

Durante este proceso, se evidencian prácticas pedagógicas cooperativas que van desapareciendo en beneficio de tecnologías de clasificación y dispositivos competitivos. Por ejemplo, un taller de escritura de textos libres, proveniente del dispositivo identitario, que favorecía la creatividad de los 
niños y luego su publicación cooperativa. Permitía trabajar las habilidades de lectura y escritura, imaginación y gramática, atención oral y concentración. Con el horizonte del SIMCE, la opción pedagógica se orienta hacia técnicas conductistas formalizadas en un "plan lector" propuesto por la Asesoría Técnica Educativa (ATE), organismo privado externo contratado en el marco de la SEP. Se instaura la evaluación regular de los niños, y se instala en el aula un "panel de lectura" decorado con colores, como una ascensión hacia las estrellas. Se fabrican etiquetas de astronautas con la foto de cada niño, y se van clasificando en función de sus resultados en la última prueba. Este panel termina ocupando el espacio central en el muro, simbolizando visualmente la competencia entre los niños y estigmatizando a los que se quedan en los niveles inferiores. Los mismos docentes se dan cuenta cómo el dispositivo tecnocrático va transformando sus prácticas hacia la puesta en competencia de los niños, generando contradicciones éticas y pedagógicas profundas. Pero la exigencia de clasificación por la Agencia de Calidad es tan imperante que no pueden permitirse resistirle, tal como lo expresan durante la exposición de los presentes hallazgos.

\section{Discusión y conclusiones}

Se ha realizado una etnografía que ha permitido evidenciar dinámicas propias a las escuelas subvencionadas chilenas ubicadas en contextos marginalizados, constreñidas por las nuevas políticas de rendición de cuentas. Comprendiendo la institución escolar como una organización inserta en un contexto político y socio-cultural, se ha develado una micro-política de la escuela atravesada por tres dispositivos: identitario, tradicional y tecnocrático. En el interior de la escuela, estos dispositivos configuran aplicaciones locales de poder, que son resistidas o apropiadas por los sujetos escolares. En este estudio, se ha observado una tensión entre por un lado una finalidad ética compartida entre los adultos involucrados en la escuela, a menudo desde una perspectiva afectiva y emancipadora; y por otro lado los imperativos ligados al sistema escolar y a su gestión por los resultados, que implican un enfoque en la estandarización del proceso educativo y una pérdida de su relevancia para los niños. De la misma manera, el estudio de Assael et al. (2014) ha puesto en evidencia los efectos de las políticas de rendición de cuentas y las transformaciones de la cultura escolar, con base en un trabajo etnográfi- 
co en las reuniones de equipo. Rojas y Leyton (2014), a partir de sus entrevistas, han traído a la luz los cambios en las prácticas educativas y la construcción de nuevas subjetividades docentes con el cambio de paradigma que implica la rendición de cuentas. Lo mismo ha revelado el estudio etnográfico y las entrevistas de Falabella (2014), en lo que analiza como "escuelas performativas". Estas producen sujetos tensionados entre sus valores propios y las exigencias del sistema educativo, que son incorporadas por los docentes.

En otro nivel, Luna (2015) ha indagado de manera etnográfica las experiencias infantiles, en escuelas municipales precarizadas y tensionadas por la exigencia de calidad, al igual que las escuelas subvencionadas. Evidencia las experiencias cotidianas que contribuyen en excluir a los sujetos que no se conforman con el marco escolar. El presente estudio confirma los hallazgos de estas investigaciones post-Ley SEP (2008), y además integra el nuevo marco regulatorio y discursivo que plantea un objetivo de inclusión social para la escuela (2015). ¿Cómo conciliar entonces estandarización e inclusión, doble exigencia normativa? En esta micro-política de la escuela, los dispositivos en disputa se configuran como maneras de decir y de actuar disponibles para los sujetos escolares, los que toman decisiones pedagógicas centrales en la experiencia escolar de los niños. Se ha evidenciado el conflicto entre lógicas de inclusión y lógicas de competencia, donde las dinámicas tecnocráticas se sobreponen finalmente sobre las dinámicas propiamente educativas.

Volviendo a la Ley de Inclusión (2015), que busca promover la mixtura social de los estudiantes, se puede cuestionar cómo puede la escuela revertir las políticas de segregación urbana. ¿Cambiará la composición del alumnado en la escuela "Estrella" con la implementación del nuevo sistema de admisión? Pensando en las nuevas configuraciones demográficas generadas por la llegada de migrantes en los sectores marginalizados, se pueden plantear otras pistas de investigación. ¿Cómo se transforma o se renueva la cultura de la escuela? ¿Cómo se incorporan los saberes de los niños migrantes que llegan con experiencias propias? ¿Cómo se rediseña el discurso nacionalista? y ¿Cómo se relaciona la acogida de los migrantes con la componente Mapuche del estudiantado? Estas líneas de investigación podrían prolongar el trabajo realizado, siempre inacabado, considerando las transformaciones permanentes que atraviesan las sociedades. 


\section{Bibliografía}

Adams St. Pierre, E. (1997). Methodology in the fold and the irruption of transgressive data. International Journal of Qualitative Studies in Education, 10(2), 175-189. doi: https://doi.org/10.1080/095183997237278

Adams St. Pierre, E. (2014). A Brief and Personal History of Post Qualitative Research: Toward "Post Inquiry". Journal of Curriculum Theorizing, 30(2), 2-19.

Adams St. Pierre, E. (2017). Writing Post Qualitative Inquiry. Qualitative Inquiry, 1-6. doi: https://doi.org/10.1177/1077800417734567

Adams St.Pierre, E., Jackson, A.\& Mazzei,L. (2016). New Empiricisms and New Materialisms: Conditions for New Inquiry. Cultural Studies. Critical Methodologies, 16(2), 99-110. doi: https://doi.org/10.1177/1532708616638694

Apablaza, M. (2015). El orden en la producción de conocimiento: Normatividades en la educación chilena en torno a diversidad. Estudios Pedagógicos, 41( $\mathrm{n}^{\circ}$ Especial), 253-266.

Armijo-Cabrera, M. (2018). Deconstruyendo la noción de inclusión: un análisis de investigaciones, políticas y prácticas en educación. Revista Electrónica Educare, 22(3), 1-26. doi: http://dx.doi.org/10.15359/ree.22-3.8

Assael, J., Acuña, F., Contreras, P. \& Corbalán, F. (2014). Transformaciones en la cultura escolar en el marco de la implementación de políticas de accountability en Chile: Un estudio etnográfico en dos escuelas clasificadas en recuperación. Estudios pedagógicos, 40(2), 07-26. doi: https://doi.org/10.4067/ S0718-07052014000300001

Ball, S. J. (1987). The micro-politics of the school: Towards a theory of school organization. London: Methue.

Ball, S. J. (2012). Foucault, Power and Education. New York: Routledge.

Bellei, C. (2013). El estudio de la segregación socioeconómica y académica de la educación chilena. Estudios Pedagógicos, 39(1), 325-345.

Blackman, S. (2007). 'Hidden Ethnography': Crossing Emotional Borders in Qualitative Accounts of Young People's Lives. Sociology, 41(4), 699-716. doi: https://doi.org/10.1177/0038038507078925

Casassus, J. (2010). Las reformas basadas en estándares: un camino equivocado. Educere et Educare. Revista de Educação, 5(9), 85-107.

De la Rosa, C. (2015). ¿Quién es el otro?: Un análisis de los discursos de diversidad en estudios sobre formación docente en Chile. Pensamiento Educativo. Revista de Investigación Educacional Latinoamericana, 52(2), 136-150. doi: https://doi.org/10.7764/PEL.52.2.2015.8 
Decreto 170 (2009). Fija normas para determinar los alumnos con necesidades educativas especiales que séran beneficiarios de las subvenciones para educación especial. Biblioteca del Congreso Nacional de Chile. Recuperado de https://goo.gl/uzoRJf

Decreto con Fuerza de Ley 1-3063 (1980). Reglamenta aplicación inciso segundo del artículo $38^{\circ}$ del DL. $n^{\circ} 3.063$, de 1979. Biblioteca del Congreso Nacional de Chile. Recuperado de https://goo.gl/bYKgX2

Decreto Ley 3.476 (1980). Fija normas a los establecimientos de enseñanza particular subvencionados por el Estado. Biblioteca del Congreso Nacional de Chile. Recuperado de https://goo.gl/oWWTPF

Ducci, M.E. (1997). Chile: el lado oscuro de una política de vivienda exitosa. EURE, Revista Latinoamericana de Estudios Urbanos y Regionales, 23(69), 99-115.

Dussel, I. (2004). Inclusión y exclusión en la escuela moderna argentina: una perspectiva postestructuralista. Cuadernos de Pesquisa, 34(122), 305-335.

Erickson, F. (1984). What Makes School Ethnography 'Ethnographic'? Anthropology \& Education Quarterly,15(1), 51-66.

Falabella, A. (2014). The Performing School: The Effects of Market \& Accountability Policies. Education Policy Analysis Archives, 22(70), 1-25.

Falabella,A. (2015). El mercado escolar en Chile y el surgimiento de la Nueva Gestión Pública: El tejido de la política entre la dictadura neoliberal y los gobiernos de la centroizquierda (1979 a 2009). Educação \& Sociedade, 36(132).

Foucault, M. (1982). The Subject and Power. Critical Inquiry, 8(4), 777-795.

Freire, P. (1970). Pedagogía del oprimido. Uruguay: Tierra Nueva.

Gallant, M. (2008). Using an ethnographic case study approach to identify sociocultural discourse. A feminist post-structural view. Education, Business and Society: Contemporary Middle Eastern Issues, 1(4), 244-254. doi: https://doi.org/10.1108/17537980810929966

Graham, L., \& Slee, R. (2008). An illusory interiority: Interrogating the discourse/s of inclusion. Educational Philosophy and Theory, 40(2), 277-293.

Guber, R. (2011). La etnografía. Método, campo y reflexividad. Buenos Aires: Siglo Veintiuno Editores.

Gurovich, A. (1999). Una ciudad interminable: La Pintana. Revista de Urbanismo [En línea], 1, 1-10. Venezuela. ISSN: 2443-468X.

Ley 20.529 (2011). Sistema Nacional de Aseguramiento de la Calidad de la Educación Parvularia, Básica y Media y su fiscalización (SNAC). Biblioteca del Congreso Nacional de Chile. Recuperado de https://goo.gl/SGQjkq 
Ley de Inclusión Escolar n 20.845 (2015). De inclusión escolar que regula la admisión de los y las estudiantes, elimina el financiamiento compartido y prohíbe el lucro en establecimientos educacionales que reciben aportes del Estado. Biblioteca del Congreso Nacional de Chile. Recuperado de https://goo.gl/krRUQ6

Ley de Subvención Escolar Preferencial, nº 20.248 (2008). Diario Oficial, Santiago, $1^{\circ}$ de Febrero. Biblioteca del Congreso Nacional de Chile. Recuperado de https://goo.gl/tYJru5

Ley General de Educación, no 20.370 (2009). Diario Oficial, Santiago, 2 de abril. Biblioteca del Congreso Nacional de Chile. Recuperado de https://goo. $\mathrm{gl} / \mathrm{uLCqwx}$

Lindblad, S. \& Popkewitz, T. (2001). Education Governance and Social Integration and Exclusion: Studies in the powers of reason and the reasons of power. Uppsala Reports on Education, 39.

Luna, L. (2015). Construyendo "la identidad del excluido": etnografía del aprendizaje situado de los niños en una escuela básica municipal de Chile. Estudios pedagógicos, 41 ( $\mathrm{n}^{\circ}$ Especial), 97-114.

Matus, C. \& Infante, M. (2011). Undoing diversity: knowledge and neoliberal discourses in colleges of education. Discourse: Studies in the Cultural Politics of Education, 32(3), 293-307.

Morales, E. \& Rojas, S. (1986). Relocalización socio espacial de la pobreza. Política estatal y presión popular, 1979-1985. (Documento de trabajo 280). Santiago: Facultad Latinoamericana de Ciencias Sociales (FLACSO).

Peña, M. (2013). Análisis crítico de discurso del Decreto 170 de Subvención Diferenciada para Necesidades Educativas Especiales: El diagnóstico como herramienta de gestión. Psicoperspectivas, 12(2), 93-103.

Pink, S. (2001). Doing Visual Ethnography: Images, Media and Representation In Research. London, UK: Sage publications.

Richardson, L. (2000). New Writing Practices in Qualitative Research. Sociology of Sport Journal, 17, 5-20.

Rockwell, E. (2009). La experiencia etnográfica. Historia y cultura en los procesos educativos. Buenos Aires: Paidós.

Rojas, M.T. \& Armijo, M. (2016). Qué es la inclusión escolar: distintas perspectivas en debate. Cuadernos de Educación, 75. Chile: Universidad Alberto Hurtado.

Rojas, M.T. \& Leyton, D. (2014). La nueva subjetividad docente. Construcción de subjetividades docentes en los inicios de la implementación de la Sub- 
vención Escolar Preferencial en Chile. Estudios pedagógicos, 40 (nº especial), 205-221.

Rojas, M.T., Falabella, A. \& Leyton, D. (2016). Madres de clases medias frente al mercado educativo en Chile: Decisiones y dilemas. En J. Corvalán, J.E. García-Huidobro y A. Carrasco (Eds.), La organización de mercado del sistema escolar chileno: Libertad, diversidad y desigualdad (pp. 231264). Chile: UC.

Serra, C. (2004). Etnografía escolar, etnografía de la educación. Revista de Educación, 334, 165-176.

Silva, V. \& Gallardo, J. (2006). Funcionamiento de familias de adolescentes (Tesis para optar al grado de licenciado en Trabajo social). Santiago de Chile: Universidad Academia de Humanismo Cristiano.

Slachevsky, N. (2015). Una revolución neoliberal: la política educacional en Chile desde la dictadura militar. Educ. Pesqui., 41 (nespecial), 1473-1486. doi: http://dx.doi.org/10.1590/S1517-9702201508141660

Valenzuela, J. P., Bellei, C., \& De los Rios, D. (2014). Socioeconomic school segregation in a marketoriented educational system. The case of Chile. Journal of Education Policy, 29(2), 217-241. doi: https://doi.org/10.1080/02 680939.2013.806995

Walsh, C. (2012). Interculturalidad y (de)colonialidad: perspectivas críticas y políticas. Revista Visao Global, 15(1-2), 61-74. ISSN: 2179-4944.

Fecha de recepción: 2018/II/29; Fecha de aceptación: 2019/01/18;

Fecha de publicación: 2018/03/01 